\title{
Medidor de Peak-flow: técnica de manejo y utilidad en Atención Primaria
}

\author{
J. Miquel-Gomara Perelló, M. Román Rodríguez* y Grupo de respiratorio \\ de la Societat Balear de Medicina Familiar I Comunitaria** \\ Médico de Familia. Centro de Salud Sineu. Mallorca. \\ * Medico de Familia. Centro de Salud San Pisá. Mallorca
}

\section{RESUMEN}

La medida del flujo espiratorio máximo (FEM) - peak expiratory flow (PEF) en terminología an glosajona - mediante medidores portátiles, consti tuye una de las herramientas más útiles y accesi bles para la valoración de la función pulmonar de asmáticos en las consultas de Atención Primaria. Tras definir el FEM y revisar las características de los medidores y las ventajas e inconvenientes de esta técnica en relación con la espirometría forza $d a$, se discuten posteriormente las indicaciones pa ra su uso con fines diagnósticos, de control evoluti vo a corto y largo plazo y para crisis asmáticas.

Palabras clave: Asma. Flujo expiratorio máxi mo (FEM). Atención Primaria.
Peak-flow meter: technique and utilities in Pri mary Health Care

\begin{abstract}
The measurement of peak expiratory flow (PEF) using portable devices, is one of the more accessi ble tools in general practice for testing pulmonary function in asthmatics. We define the PEF and we review the peak flowmeters' technical characteris tics and their advantages and disadvantages com paring to spirometers, then we discuss the indica tions for using PEF measurement for diagnosis, short and long monitoring and crisis control.
\end{abstract}

Key words: Asthma. Peak expiratory flow rate. Primary Health Care.

\section{INTRODUCCIÓN}

Estudios poblaciones realizados en el territorio español aportan datos que sitúan al asma como una de las enfermedades crónicas más prevalentes sobre todo en grupos de edad infantil y juvenil ${ }^{1}$. En los últimos años hemos asistido a avances en el conocimiento de los mecanismos fisiopatológicos del asma, considerándose una enfermedad inflamatoria bronquial, y en su tratamiento, con la aparición de nuevos fármacos y métodos de inhalación.
Estos avances no se han acompañado de nuevos métodos diagnósticos que expresen objetivamente el grado de inflamación y, aunque existen marcadores específicos, su medición queda aún lejos de la práctica diaria de las consultas. El diagnóstico del paciente asmático se sigue basando en la clínica y en las pruebas de función respiratoria, que valoran las características funcionales del paciente (obstrucción, reversibilidad, hiperreactividad bronquial, y variabilidad) como estimación indirecta del grado de inflamación.

**Grupo de Respiratorio de la Societat Balear de Medicina Familiar i Comunitaria: Amalia Gómez Nadal, Verónica Lluch Garbí, Margalida Mut Tomás.

Aceptación: 27-11-01 
El flujo espiratorio máximo (FEM) o peak expira tory flow (PEF) en terminología anglosajona, es el mayor flujo que se alcanza durante una maniobra de espiración forzada. Se consigue al haber espirado el $75-80 \%$ de la capacidad pulmonar total (dentro de los primeros $100 \mathrm{~ms}$ de espiración forzada) y se expresa en litros/minuto, litros/segundo o como porcentaje de su valor de referencia. Refleja el estado de las vías aéreas de gran calibre ${ }^{2}$, y es un índice aceptado como medida independiente de la función pulmonar ${ }^{3}$.

Los espirómetros actuales ofrecen los valores de FEM junto al resto de parámetros de función pulmonar. No obstante, la utilidad del FEM se centra en la posibilidad de medición en distintas circunstancias mediante medidores portátiles. La ventaja de éstos es que pueden ser transportados y manejados por el paciente de manera sencilla.

\section{APARATOS MEDIDORES DE FEM (mFEM)}

Se trata de aparatos, generalmente tubos, que en su interior presentan un mecanismo de pistón-muelle o de aspa que se mueve al aplicar un flujo de aire durante una maniobra de espiración forzada. Una vez se alcanza el máximo, un indicador fija el resultado en una escala de litros por minuto impresa en el tubo.

Existen varios tipos de medidores en el mercado español (Tabla I).

\section{Tabla I}

MEDIDORES DE FLUJO ESPIRATORIO EXISTENTES EN EL MERCADO ESPAÑOL

F5 PROFAS S.L.

Asmaplan® adultos peakflow. C.N. 169532

Asmaplan ${ }^{\circledR}$ niños peakflow. C.N. 169540.

BOEHRINGER INGELHEIM

Mini-bell囚 flujo bajo peakflow. C.N. 259127.

Mini-bell囚 peakflow. C.N. 338665.

BRIGHTON MEDICAL

Miniwright $₫$ adulto peakflow. C.N. 332437.

Miniwright ${ }^{\circledR}$ AFS low pediátrico peakflow. C.N. 332445.

ALDO-UNION S.A.

Personal best ${ }^{\circledR}$ escala normal peakflow. C.N. 376814.

Personal best ${ }^{\circledR}$ escala reducida peakflow. C. N. 376822.

CBF LETI S.A.

PF control ${ }^{\circledR}$ adulto peakflow. C.N. 175927.

PF control ${ }^{\circledR}$ bajo flujo peakflow. C.N. 175919.

PF control ${ }^{\circledR}$ niños peakflow. C.N. 175935.

MERCK F. Y Q

Astech peak flow meter ${ }^{\circledR}$. C.N. 361840.

C.N.: Código Nacional
La American Thoracic Society, dentro de su normativa sobre espirometría, ha recomendado unos estándares de funcionamiento para estos aparatos ${ }^{4}$ :

1. Precisión de los flujos entre 0 y 900 l/min (0 a $15 \mathrm{l} / \mathrm{s}$ ) ,dando lecturas dentro del $10 \%$ o de $10 \mathrm{l} / \mathrm{min}$ del verdadero valor medido mediante espirómetro.

2. Repetitibilidad: la diferencia entre dos maniobras no debe superar el $3 \%$ o 10 1/min.

3. Reproducibilidad: la variabilidad entre los aparatos debe ser menor del 5\% o 20 1/min.

Existen medidores portátiles electrónicos. Entre sus ventajas, obvian la necesidad de registro manual de los valores por parte del paciente, aumentan la exactitud del registro y graban el momento del día en que se realiza la maniobra. Algunos pueden incluso medir valores de volumen expiratorio forzado en el primer segundo (FEV1) y capacidad vital forzada (FVC). Su principal inconveniente es su alto precio.

Muchos medidores no adjuntan información sobre su vida media o de su calibración, que debe realizarse con descompresor explosivo según un estándar. No se dispone de un dispositivo comercializado que permita un control de calidad periódico. Se recomienda la validación de la exactitud del mFEM frente a una espirometría de laboratorio al menos una vez al año y siempre que surjan dudas sobre la validez de las lecturas del FEM ${ }^{5}$.

Si es el paciente el que posee el medidor, debe revisarse el funcionamiento del aparato en cada visita de seguimiento.

No hay consenso sobre la periodicidad con que un medidor de FEM debe ser cambiado. No se han observado diferencias significativas en la precisión de la medida tras un año de uso ${ }^{6}$. Otro estudio reciente demostraba la fiabilidad de los medidores tipo mini-Wright a lo largo de 5 años, después de ser usado en mas de 2.000 ocasiones y restringir su recambio a casos de obvio mal funcionamiento ${ }^{7}$. Existe acuerdo generalizado de que al recambiar un medidor debe hacerse por otro del mismo fabricante ya que puede darse una escasa concordancia entre las diferentes marcas ${ }^{8,9}$.

\section{TÉCNICA DE MEDICIÓN}

Para un correcto registro del FEM es necesario adiestrar al paciente en el uso del medidor especificando cada uno de los pasos a seguir (Tabla II).

\section{TABLAS DE REFERENCIA}

Los valores de normalidad se determinan por talla, edad y sexo. Existen varias formas de presentación de los mismos (Fig. 1) (Tabla III). Aunque existen pocos estudios sobre valores de referencia de FEM en población general, cada comunidad de- 


\section{Tabla II}

TÉCNICA DE MEDICIÓN DE FEM

\section{Posición de pie.}

Colocar el indicador a cero.

Sujetar el medidor en posición horizontal sin interferir el recorrido del indicador.

Efectuar una inspiración máxima.

Cerrar los labios alrededor de la boquilla.

Evitar bloquear la salida de aire con la lengua.

Soplar de forma explosiva, lo mas rápido y fuerte posible.

Realizar la lectura y anotar su valor.

Colocar el indicador a cero.

Repetir el proceso dos veces más y registrar su valor más alto.

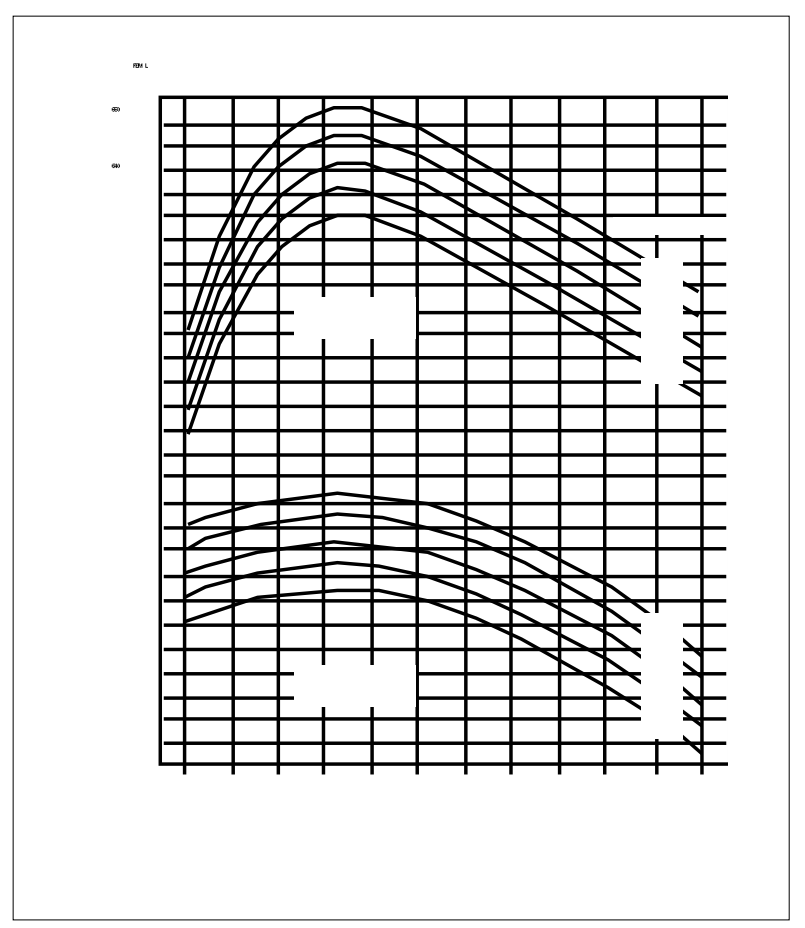

Figura 1

Valores de normalidad del flujo respiratorio máximo en adultos

bería manejar sus propias tablas de referencia o las obtenidas en poblaciones similares ${ }^{2}$.

\section{VENTAJAS Y LIMITACIONES DE LA MEDICIÓN DEL FLUJO PICO}

\section{Ventajas}

1. Los resultados de la medida del FEM se correlacionan con los valores de FEV $1^{3,10-12}$ y proporcionan una estimación del grado de obstrucción bronquial.

2. Fatiga menos que la espirometría forzada ya que no requiere una espiración completa hasta vo- lumen residual, maniobra que en algunos pacientes desencadena tos o sibilancias.

3. El medidor es pequeño, portátil y de uso sencillo, lo que permite mediciones en distintos entornos del paciente. Es relativamente fácil obtener la colaboración precisa por parte del enfermo. Puede ser utilizado a partir de los 5-6 años de edad.

4. El mantenimiento técnico del aparato es mínimo.

5. La interpretación del resultado es simple al contrario de lo que puede suceder con la espirometría. Ello permite que el personal sanitario adiestre al paciente para su interpretación.

\section{Inconvenientes}

1. No puede sustituir a la espirometría cuando se usa para la evaluación inicial del paciente asmático ${ }^{5}$. La sensibilidad del FEM es menor que la del FEV1. Se han detectado sobrelecturas de hasta $80 \mathrm{l} / \mathrm{min}$ en rangos medios (aproximadamente $300 \mathrm{l} / \mathrm{min}$ ), e infralecturas de hasta $60 \mathrm{l} / \mathrm{min}$ en rangos altos (aproximadamente $600-800 \mathrm{l} / \mathrm{min})^{13}$.

2. No proporciona información de la función de las vías de pequeño calibre.

3. Al contrario de la espirometría, no es útil en el abordaje de enfermos con enfermedad pulmonar obstructiva crónica (EPOC).

4. Al ser dependiente del esfuerzo y de una correcta técnica de realización, puede ser menos vaporable en niños pequeños y ancianos, y susceptible de simulación.

5. En seguimiento a largo plazo requiere el compromiso del paciente para realizar la maniobra con la periodicidad aconsejada y registrar los datos, lo cual implica un esfuerzo, especialmente en fases estables de la enfermedad.

\section{¿CUÁNDO MONITORIZAR EL PICO FLUJO? USO CLÍNICO}

El médico de familia se puede beneficiar de la medida de los valores del FEM mediante aparatos portátiles en diversas situaciones. A continuación veremos sus principales indicaciones tanto en el abordaje diagnóstico, como en el seguimiento del paciente asmático (Tabla IV).

\section{UTILIDAD DIAGNÓSTICA}

\section{Estudio de variabilidad}

Los valores de FEM están sometidos a cambios circadianos tanto en individuos sanos como 
Tabla III

INTERPRETACIÓN DEL PEAK-FLOW. VALORES NORMALES TEÓRICOS DEL FLUJO RESPIRATORIO PICO (LITROS/MIN)

Hombre. Desviación normal 48 I/min

\begin{tabular}{lcccccccccccc}
\hline $\begin{array}{l}\text { Edad/ } \\
\text { Altura }\end{array}$ & $\begin{array}{c}15 \\
\text { años }\end{array}$ & $\begin{array}{c}20 \\
\text { años }\end{array}$ & $\begin{array}{c}25 \\
\text { años }\end{array}$ & $\begin{array}{c}30 \\
\text { años }\end{array}$ & $\begin{array}{c}35 \\
\text { años }\end{array}$ & $\begin{array}{c}40 \\
\text { años }\end{array}$ & $\begin{array}{c}45 \\
\text { años }\end{array}$ & $\begin{array}{c}50 \\
\text { años }\end{array}$ & $\begin{array}{c}55 \\
\text { años }\end{array}$ & $\begin{array}{c}60 \\
\text { años }\end{array}$ & $\begin{array}{c}65 \\
\text { años }\end{array}$ & $\begin{array}{c}70 \\
\text { años }\end{array}$ \\
\hline $160 \mathrm{~cm}$ & 518 & 568 & 598 & 612 & 613 & 606 & 592 & 578 & 565 & 555 & 544 & 534 \\
$168 \mathrm{~cm}$ & 530 & 580 & 610 & 623 & 623 & 617 & 603 & 589 & 577 & 566 & 556 & 546 \\
$175 \mathrm{~cm}$ & 540 & 590 & 622 & 636 & 635 & 627 & 615 & 601 & 588 & 578 & 568 & 558 \\
$183 \mathrm{~cm}$ & 552 & 601 & 632 & 645 & 646 & 638 & 626 & 612 & 600 & 589 & 578 & 568 \\
$190 \mathrm{~cm}$ & 562 & 612 & 643 & 656 & 656 & 649 & 637 & 623 & 611 & 599 & 589 & 579 \\
\hline
\end{tabular}

Mujeres. Desviación normal 42 1/min

\begin{tabular}{|c|c|c|c|c|c|c|c|c|c|c|c|c|}
\hline $\begin{array}{l}\text { Edad/ } \\
\text { Altura }\end{array}$ & $\begin{array}{c}15 \\
\text { años }\end{array}$ & $\begin{array}{c}20 \\
\text { años }\end{array}$ & $\begin{array}{c}25 \\
\text { años }\end{array}$ & $\begin{array}{c}30 \\
\text { años }\end{array}$ & $\begin{array}{c}35 \\
\text { años }\end{array}$ & $\begin{array}{c}40 \\
\text { años }\end{array}$ & $\begin{array}{c}45 \\
\text { años }\end{array}$ & $\begin{array}{c}50 \\
\text { años }\end{array}$ & $\begin{array}{c}55 \\
\text { años }\end{array}$ & $\begin{array}{c}60 \\
\text { años }\end{array}$ & $\begin{array}{c}65 \\
\text { años }\end{array}$ & $\begin{array}{c}70 \\
\text { años }\end{array}$ \\
\hline $\begin{array}{l}145 \mathrm{~cm} \\
152 \mathrm{~cm} \\
160 \mathrm{~cm} \\
168 \mathrm{~cm} \\
175 \mathrm{~cm}\end{array}$ & $\begin{array}{l}438 \\
450 \\
461 \\
471 \\
481\end{array}$ & $\begin{array}{l}445 \\
456 \\
467 \\
478 \\
488\end{array}$ & $\begin{array}{l}450 \\
461 \\
471 \\
482 \\
493\end{array}$ & $\begin{array}{l}452 \\
463 \\
474 \\
485 \\
496\end{array}$ & $\begin{array}{l}452 \\
463 \\
473 \\
484 \\
496\end{array}$ & $\begin{array}{l}449 \\
460 \\
470 \\
482 \\
493\end{array}$ & $\begin{array}{l}444 \\
456 \\
467 \\
478 \\
488\end{array}$ & $\begin{array}{l}436 \\
448 \\
458 \\
470 \\
480\end{array}$ & $\begin{array}{l}426 \\
437 \\
449 \\
460 \\
471\end{array}$ & $\begin{array}{l}415 \\
425 \\
437 \\
448 \\
458\end{array}$ & $\begin{array}{l}400 \\
410 \\
422 \\
434 \\
445\end{array}$ & $\begin{array}{l}385 \\
396 \\
407 \\
418 \\
428\end{array}$ \\
\hline
\end{tabular}

Niños. menores de 15 años

\begin{tabular}{|c|c|c|c|c|c|c|c|c|c|c|c|c|}
\hline \multirow[t]{2}{*}{ Altura } & 91 & 99 & 107 & 114 & 122 & 130 & 137 & 145 & 152 & 160 & 168 & 175 \\
\hline & $\mathrm{cm}$ & $\mathrm{cm}$ & $\mathrm{cm}$ & $\mathrm{cm}$ & $\mathrm{cm}$ & $\mathrm{cm}$ & $\mathrm{cm}$ & $\mathrm{cm}$ & $\mathrm{cm}$ & $\mathrm{cm}$ & $\mathrm{cm}$ & $\mathrm{cm}$ \\
\hline & 100 & 120 & 140 & 170 & 210 & 250 & 285 & 325 & 360 & 400 & 440 & 480 \\
\hline
\end{tabular}

Tomado de "Guía semFYC de actuación en Atención Primaria.

en asmáticos ${ }^{14}$. El pico flujo es máximo alrededor de las cuatro de la tarde alcanzando los valores más bajos cerca de las cuatro de la madrugada. No se ha establecido el mecanismo exacto que explique estas variaciones en la resistencia de las vías aéreas. Se ha implicado un aumento en la hiperreactividad inespecífica de la vía aérea y del tono parasimpático nocturno, y otros cambios bioquímicos. El mínimo del FEM coincide con el mayor valor plasmático de la histamina y el mínimo de AMPc y epinefrina; los niveles de cortisol plasmático se alcanzan alrededor de la mediano- che, y ello no parece diferir entre asmáticos y no asmáticos ${ }^{15}$.

Sin embargo, la variabilidad del FEM en pacientes asmáticos está incrementada comparada con sujetos normales. El promedio de la variación del FEM en sujetos normales se situa en torno al 8,3\%, mientras que en los pacientes asmáticos puede llegar a alcanzar el $50 \%^{14}$.

La variabilidad de los parámetros de función pulmonar como indicadores de hiperreactividad bronquial se ha relacionado con el grado de inflamación de la vía aérea en asmáticos, sin embargo se requie-

UTILIDADES DEL MEDIDOR DE PICO-FLUJO

\begin{tabular}{lll} 
Diagnóstico & & \multicolumn{1}{c}{ Seguimiento } \\
& corto plazo & Iargo plazo \\
\hline Estudio de variabilidad & Clasificación & Autocontrol asmático \\
Estudio de asma laboral & Establecer M M P* \\
Reversibilidad (prueba broncodilatadora) & Asma de difícil control \\
Estudio de asma de esfuerzo & Control terapéutico
\end{tabular}

Valoración de la gravedad de las crisis

*MMP: mejor marca personal 
ren estudios que relacionen la variación del FEM con marcadores directos de inflamación antes de una conclusión definitiva sobre su uso como medida de inflamación bronquial ${ }^{5,16}$. No se ha podido demostrar por el momento una relación entre el grado de inflamación bronquial y la variabilidad del $\mathrm{FEM}^{17}$.

Para el cálculo de variabilidad se registran los valores de FEM medidos a primera hora de la mañana y a última hora de la tarde durante un periodo de tiempo que habitualmente se establece en dos semanas y se realiza un cálculo de la diferencia entre dichos valores ${ }^{18}$. No existe consenso sobre la fórmula a utilizar para medir la variabilidad y se han aplicado varias. Destacamos las más útiles en la práctica clínica (Tabla V).

Una cifra de variabilidad mayor del $20 \%$ es sugestiva y para muchos autores diagnóstica de asma ${ }^{19}$.

Su aplicabilidad diagnóstica se centra en aquellos casos con alta sospecha clínica de asma en los que la realización de una espirometría forzada con PBD no ha confirmado el diagnóstico.

Hay asmáticos que presentan patrones característicos de FEM diario con una alta variabilidad. Estos casos suelen ser de difícil control y de riesgo elevado (mayor incidencia de muerte súbita) ${ }^{20}$.

1. Patrón lábil o frágil (brittle asthma). Se caracteriza por oscilaciones profundas del flujo espiratorio durante la jornada, sin seguir ritmo alguno que lo haga previsible.

2. Patrón matinal (morning dipper). Se caracteriza por presentar flujos normales o aceptables durante el día con caída de los mismos a primera hora de la mañana. Este descenso matutino es especialmente intenso en pacientes con broncoespasmo nocturno.

\section{Estudio de asma profesional}

Debemos tener en cuenta la posibilidad de una exposición laboral de riesgo ante cualquier enfermo asmático ya que un diagnóstico tardío empeorará, en la mayoría de los casos, el pronóstico.

La exploración más adecuada para el estudio funcional del paciente con sospecha de asma ocupacional es la medida del FEM con un medidor portátil dentro y fuera del trabajo. Es necesario registrar el FEM cuatro o más veces al día, durante un tiempo mínimo de tres semanas con actividad laboral habitual y una semana de baja laboral. Los

\section{Tabla V}

MEDICIÓN DE VARIABILIDAD

FEM máximo-FEM mínimo / FEM máximo

FEM máximo-FEM mínimo / (FEM máximo + FEM mínimo)/ 2 datos obtenidos permitirán valorar la relación entre el deterioro de la función pulmonar y la actividad laboral: detección de las caídas del FEM asociadas al trabajo (inmediatas y retardadas), deterioro progresivo a lo largo de la semana, y el incremento de los valores fuera del trabajo. Variaciones superiores al $20 \%$ se consideran relevantes ${ }^{21}$.

Estas medidas del FEM deben complementarse con otros estudios para la confirmación diagnóstica, que habitualmente se realizan en el ámbito de consulta especializada.

\section{Estudio de asma de esfuerzo}

Ante la posibilidad de asma relacionada con el ejercicio, se plantea la realización de un test de carrera libre, que en caso de no disponer de espirómetro, se puede realizar mediante un medidor de FEM.

Se realiza una medición en reposo y posteriormente, tras unos minutos de ejercicio en los que el paciente debe alcanzar el $80 \%$ de la frecuencia cardiaca submáxima (220 pulsaciones por minuto menos la edad), se efectuaran mediciones consecutivas del FEM cada 5 minutos durante 20-30 minutos. Son significativas caídas de FEM superiores al $15 \%$ en cualquiera de las mediciones ${ }^{22}$. En pacientes adultos es recomendable la realización de la prueba bajo monitorización cardiorrespiratoria.

\section{Demostración de reversibilidad}

Los medidores portátiles se pueden utilizar de manera similar a la espirometría forzada. Se trata de valorar el posible cambio en las cifras de FEM con la administración de un broncodilatador. Tras la medición del FEM basal, se efectúa una prueba broncodilatadora (PBD) con la administración de $400 \mu \mathrm{g}$ de salbutamol y se repite la determinación del FEM 15-20 minutos después. Se considera un test positivo si el incremento del FEM es superior al $15 \%$.

PBD: FEM post - FEM pre x 100 / FEM pre ${ }^{23}$.

\section{UTILIDAD EN SEGUIMIENTO}

Las cifras del FEM junto a la valoración subjetiva de los síntomas y la medicación broncodilatadora requerida forman el trípode en que se asienta el control clínico ambulatorio del enfermo asmático. El personal sanitario debe promover el uso del medidor con una frecuencia adaptada al curso de la enfermedad. El registro del FEM nos permite la medida objetiva del estado funcional ambulatoriamente tanto de manera puntual en las visitas de seguimiento como en el propio domicilio o trabajo del paciente. 


\section{Mejor marca personal (MMP)}

Se consideran normales aquellos valores de FEM que superen el $80 \%$ del valor teórico. No obstante, es preferible tomar como valor de referencia la mejor marca personal (MMP) obtenida por el paciente.

Para establecer la MMP se mide el FEM durante un periodo de 2-3 semanas en las mejores condiciones posibles de estabilidad. Para alcanzar esta situación, puede ser necesario un ciclo previo de corticoides orales (Tabla VI) ${ }^{19}$.

El MMP debe reevaluarse para objetivar los cambios debidos a la progresión de la enfermedad. Niños con asma moderado-severo deben repetir un ciclo corto de monitorización cada 6 meses para establecer cambios en su MMP que ocurren durante el crecimiento.

\section{Diario del asmático}

Para el autorregistro del FEM se apuntan los valores obtenidos por el propio paciente en una hoja determinada a tal efecto (diario del asmático) en la que conste además día y hora de la medición, síntomatología, pauta terapéutica y ocasionalmente algún comentario sobre posibles desencadenantes de crisis (incumplimiento terapéutico, circunstancias físicas-atmoféricas, etc.).

\section{Monitorizacion a corto plazo}

A pesar de que la indicación principal de automedición de FEM en el asma sea para largos periodos, existen varias indicaciones para realizar un registro de FEM a corto plazo durante 2-3 semanas:

-Establecer la mejor marca personal.

- Clasificar al paciente según severidad.

- Valorar posibles factores implicados en el empeoramiento.

—Detección de casos de asma inestable.

—Estudio de asma laboral.

\section{Tabla VI}

\section{OBTENCIÓN DE LA MEJOR MARCA PERSONAL (MMP)}

Paciente estabilizado y en tratamiento (posibilidad de tanda de corticoides orales)

Medición a primera hora de la tarde

Medición tras inhalación de broncodilatadores

Desconfiar de un valor aislado o de valores $<80 \%$ de referencia

Revisión periódica, sobre todo en niños

Revisión siempre tras cambio de medidor
-Disminuir la frecuencia de uso de la medicación de rescate durante periodos de empeoramiento/sintomáticos.

-Evaluar respuestas a cambios en el tratamiento de mantenimiento:

- Aumento de dosis.

- Introducción de nuevo fármaco.

- Retirada paulatina de corticoides.

- Cambio de técnica inhalatoria.

- Tratamiento de pacientes con reflujo gastroesofágico asociado.

\section{Seguimiento a largo plazo}

Para mejorar el control de la enfermedad se debe instaurar un programa integral de educación sanitaria que favorezca el máximo grado de autocontrol ${ }^{24-29}$. La automedición del FEM permite al paciente tomar decisiones apoyándose en variaciones objetivas y es fundamental para el cumplimiento de este objetivo.

Los pacientes con asma persistente moderado-severo deben aprender a monitorizar su FEM y tener un medidor de pico flujo en su domicilio. No se recomienda una monitorización a largo plazo en asma intermitente o persistente leve a menos que el paciente o el profesional lo considere útil para guiar sus decisiones terapéuticas ${ }^{29}$. Cualquier paciente que desarrolle crisis severas puede beneficiarse del registro de FEM domiciliario ${ }^{19}$ (Tabla VII).

El uso regular del mFEM puede ser de gran ayuda en asmáticos con baja percepción de la limitación al flujo aéreo. Se desconoce la magnitud del problema, el mecanismo subyacente puede representar una adaptación a la progresiva broncoconstricción. La mayoría de expertos coinciden en que la baja percepción contribuye a un control subóptimo de la enfermedad y a un retraso en solicitar atención médica 5 .

El descenso matutino del FEM es un buen indicador de la severidad del asma; puede predecir la variación diurna durante el mismo día ${ }^{10}$. Los valores reiterados de FEM menores del $80 \%$ de la MMP indican un control inadecuado y la necesidad de establecer cambios terapéuticos. En esta circunstancia se recomienda registrarlo más de una vez al día (de nuevo,

\section{Tabla VII}

\section{INDICACIONES DE AUTOMEDIDA DE FEM A LARGO PLAZO}

Asma persistente moderado-severo

Asma persistente leve para guiar decisiones terapéuticas.

Asmáticos con antecedentes de crisis severas (riesgo vital)

Asma de difícil control (morning dippers) 
antes de tomar broncodilatador). Esta recomendación no está basada en datos científicos, pero sí en la lógica de reducir retrasos en el tratamiento. Las medidas adicionales de FEM durante el día deben capacitar al paciente para detectar si su asma empeora o si mejora tras tomar la medicación. De empeorar, tendrá la oportunidad de responder rápidamente a ello con aumentos de medicación.

Cualquier paciente que desarrolle crisis severas puede beneficiarse de la monitorización del pico de flujo.

\section{Tarjeta de autocontrol (Fig. 2)}

La opinión de los grupos de expertos es que valores por debajo del $80 \%$ de la MMP indican necesidad de medicación adicional y por debajo del $50 \%$ una exacerbación severa ${ }^{19}$. Estos límites se establecen de manera arbitraria en cierta medida. El énfasis no está en el valor específico del FEM, sino más bien en la diferencia con su MMP o de una lectura con la siguiente. Debe valorarse según las necesidades individuales de los pacientes y de sus patrones de FEM.

Los puntos de corte pueden recordarse mas fácilmente mediante el sistema de zonas de las tarjetas de autocontrol ${ }^{22}$. Se trata de recomendaciones escritas entregadas al paciente con la actitud a seguir según síntomas o valores de FEM divididas en franjas de colores que se corresponden con el sentido de los colores de un semáforo.

La zona verde se caracteriza por buena capacidad funcional, con FEM superior al $80 \%$.

La franja amarilla indica precaución, incluye medidas de FEM del 50 al 80\% con o sin aparición de síntomas como sibilancias, opresión torácica, despertar nocturno. Implica el uso de medicación de rescate.

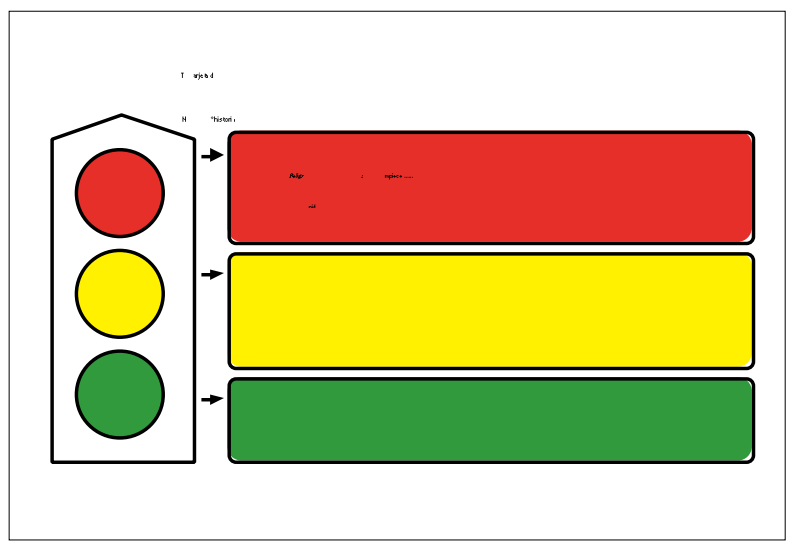

Figura 2
La zona roja corresponde a FEM inferior al $50 \%$.

Dado que la zona amarilla comprende un amplio espectro de severidad de asma, los clínicos pueden considerar recomendaciones distintas para la zona amarilla alta $(65-80 \%)$ y zona amarilla baja $(50-64 \%)$.

\section{UTILIDAD EN CRISIS}

Previamente o al inicio de la crisis se produce un descenso significativo del FEM.

La evaluación basada en la anamnesis y exploración física tiene un valor limitado para establecer la severidad de una crisis ${ }^{30}$. Así se ha demostrado en trabajos que comparan valores de FEV1 y FEM con la valoración de síntomas y signos tras una agudización. Esto sugiere la necesidad de suplementar la evaluación clínica con medidas objetivas de obstrucción al flujo aéreo en los pacientes con crisis asmática.

La monitorización del FEM en las crisis nos ayudará para:

1. Evaluación de la gravedad. El valor del FEM es una de las variables utilizadas por consenso internacional para la clasificación de las agudizaciones ${ }^{31}$.

2. Guía sobre la actitud a tomar: decisiones de tratamiento, remisión al hospital. En algoritmos de actuación referidas a áreas de urgencias hospitalarias figura el valor de FEM como variable a considerar para decidir ingreso del paciente.

3. Respuesta al tratamiento. Algunos autores le atribuyen además valor predicitivo de recaída pre$\mathrm{coz}^{32,33}$. La monitorización del FEM puede reducir la necesidad de realizar gasometría arterial en al menos el $40 \%$ de asmáticos que son tratados en urgencias por exacerbación ${ }^{34}$, particularmente en aquéllos con FEM mayor de $2001 / \mathrm{min}^{35}$. A pesar de que la monitorización del FEM se recomienda para todos los asmáticos que requieren atención en urgencias, se constata una amplia disonancia en la práctica clínica.

\section{CORRESPONDENCIA:}

Miguel Román Rodríguez

C/ Murta, 22

07193 Palmanyola. Baleares

e-mail: mroman@gapmp01.insalud.es 


\section{Bibliografía}

1. Grupo español del Estudio del Asma. Estudio Europeo del Asma: Prevalencia de síntomas relacionados con el asma en cinco areas españolas. Med Clin (Barc) 1995; 104: 487-92

2. López Guillén A, Marqués Amat L. Uso de los medidores del flujo espiratorio máximo (FEM) en el asma. Arch Bronconeumol 1994; 30: 301-6.

3. National Heart, Lung and Blood Institute. National Asthm Education Program: expert panel report: guidelines for the diagnosis and management of asthma. J Allergy Clin Immunol 1991; 88: 425-534.

4. American Thoracic Society. Standardization of spirometry, 1994 update. Am J Resp Crit Care Med 1995; 152: 1107-36.

5. Jain P, Kavuru M, Emerman C, Ahmad M. Utility of Peak Expiratory Flow Monitoring. Chest 1998; 114: 861-76.

6. Miles JF, Miller MR. The effect of one year of patient use on the accuracy of Mini Wright peak flow meters. Eur Respir J 1993; 6: 144

7. Douma WR, van der Mark TW, Folgering HT. Mini-Wright peak flow meters are realible after 5 years use. Eur Respir J 1997; 10: 457-9.

8. Hegewald MJ, Crapo RO, Jensen RL. Intraindividual peak flow variability. Chest 1995; 107: 156-61.

9. Kennedy DT, Chang Z, Small RE. Selection of peak flow meters in ambulatory asthma patients: a review of the literature. Chest 1998; 114: 587-92.

10. Mendoza G. Peak flow monitoring. J Asthma 1991; 28: 161-77.

11. Van As A. The accuracy of peak expiratory flow meters. Chest 1982; 82: 3 .

12. Hankinson JL, Filos MS, Kinsley KB, Petsonk EL. Compa ring Mini-Wright and spirometer measurements of peak expiratory flow. Chest 1995; 108: 407-10.

13. Miller MR, Dickinson SA, Hitchings DJ. The accuracy of portable peak flow meters. Thorax 1992; 47: 904-9.

14. Hetzel MR, Clark TJH. Comparison of normal and asthmatics circadians rythms in peak expiratory flow rate. Thorax 1980; 23: 219-21.

15. Smolensky MH, Barnes PJ, Reinberg A, Mc Govern JP. Chronobiology and asthma I. Day-night differences in bronchial patency and dyspnea and circadian rhythm dependencies. J Asthma 1986; 23: 321-43.

16. Boezen HM, Postma DS, Schouten JP,Kerstjens H, Rijcken B. PEF variability, bronchial responsiveness and their relation to allergy markers in a random population (20-70 yr). Am J Respir Crit Care Med 1996; 154: 30-5.

17. Gutierrez V, Prieto L, Torres V, Trenor R, Pérez C, Bertó JM, et al. Relationship betwen induced sputum cell counts anf fluid-phase eosinophil cationic protein and clinical or physiologic profiles in mild asthma. Ann Allergy Asthma Inmunol 1999; 82: 559-65.

18. Lebowitz MD. The use of peak expiratory flow rate measurements in respiratory disease. Pediatric Pulmonology 1991; 11: 166-74.

19. National Heart, Lung and Blood Institute. National Asthma
Education Program: expert panel report 2: guidelines for the diagnosis and management of asthma. Bethesda, MD: National Institutes of Health; May 1997 (NIH publication $n^{\circ}$ 97-4051).

20. Boezen HM. Schouten JP, Postma DS. Relation between respiratory symptoms, pulmonary function and peak flow variability in adults. Thorax 1995; 50: 121-6.

21. Monsó Molas E, Morera Prat J. Asma ocupacional. En: Albert Marín. Asma bronquial. Barcelona: Doyma, 1998. p. 129-37.

22. Grupo de trabajo de asma de la Sociedad Española de Medicina Familiar y Comunitaria. Recomendaciones semFYC. Asma. Barcelona: EdiDe, 1996. p. 19-34.

23. Holgate ST, Pauwels RA. Diagnosis and classification. En: Holgate ST, Pauwels RA. Fast Facts-Asthma. Oxford: Health Press Limited, 1999. p. 21-9.

24. Charlton I, Charlton G, Broomfield J, Mullee MA. Evaluation of peak flow and symptoms only self management plans for control of asthma in general practice. Br Med $\mathbf{J}$ 1990; 301: 1355-9.

25. Grampian Asthma Study of Integrated Care (GRASSIC). Efectiveness of routine self monitoring of peak flow in patients with asthma. BMJ 1994; 308: 564-7.

26. Woolcock AJ, Yan K, Salome CM. Effect of therapy on bronchial hyperresponsiveness in the long-term management of asthma. Clin Allergy 1988; 18: 165-76.

27. Ignacio-García JM, González Santos P. Asthma self-management education program by home monitoring of peak expiratory flow. Am J Respir Crit Care Med 1995; 151: 353-9.

28. Lahdensuo A, Haahtela T, Herrala J, Kava T, Kiviranta K, Kuusisto P, et al. Randomized comparison of guided selfmanagement and traditional treatment of asthma over 1 year. BMJ 1996; 312: 748-52.

29. Jones KP, Mullee MA, Middleton M, Chapman E, Holgate ST and the British Thoracic Society Research Committee. Peak flow based asthma self-management: a randomised controlled study in general practise. Thorax 1995; 50: 851-7.

30. Dales RE, Nunes F, Partyka D. Clinical Prediction of airways hiperresponsiveness. Chest 1988; 93: 984-6.

31. Cross D, Nelson HS. The role of peak flow meter in the diagnosis and management of asthma. J Allergy Clin Immunol 1991; 87: 120-8.

32. Chapman KR, Verbeek PR, White JG. Effect of a short course of prednisone in the prevention of early relapse after the emergency room treatment of acute asthma. N Engl J Med 1991; 324: 788-94

33. Emerman C, Cydulka RK. Factors associated with relapse after emergency department treatment for acute asthma. Ann Emerg Med 1995; 26: 6-11.

34. Martin TG, Elenbass RM, Pingleton SH. Use of peak expiratory flow rates to eliminate unnecessary arterail blood gases in acute asthma. Ann Emerg Med 1982; 11: 70-3.

35. Nowak RM, Tomlñanovich MC, Sarkar DD. Arterial blood gases and pulmonary function testing in acute bronchial asthma: predicting patient outcomes. JAMA 1983; 249: 2043. 